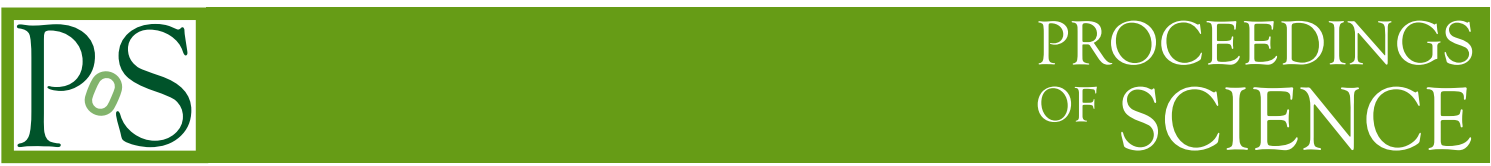

\title{
The Telescope Array Low-energy Extension
}

\author{
Shigeharu Udo*, Shoichi Ogio ${ }^{a}$, Masahiro Takeda ${ }^{b}$, Toshiyuki Nonaka $^{b}$, Hiroyuki \\ Sagawa $^{b}$, Yoshiki Tsunesada ${ }^{a}$, Shoichi Kishigami ${ }^{a}$, Ryosuke Sahara ${ }^{a}$, Yuichi \\ Takahashi $^{a}$ and the Telescope Array Collaboration \\ Faculty of Engineering, Kanagawa University \\ ${ }^{a}$ Graduate School of Science, Osaka City University \\ ${ }^{b}$ Institute for Cosmic Ray Research, University of Tokyo \\ E-mail: Shigedn.kanaqawa-u.ac.ip
}

The Telescope Array Low-energy Extension (TALE) experiment is a hybrid air shower detector for observation of air showers produced by very high energy cosmic rays above $10^{16.5} \mathrm{eV}$. TALE is located at the Telescope Array (TA) experiment site in the western desert of Utah, USA. TALE has a Surface Detector (SD) array made up of 103 scintillation counters (40 with $400 \mathrm{~m}$ spacing, 36 with $600 \mathrm{~m}$ spacing and 27 with $1.2 \mathrm{~km}$ spacing) and a Fluorescence Detector (FD) station consisting of 10 FD telescopes located at the Telescope Array Middle Drum FD station, which is made up of 14 telescopes. TALE-FD full operation started in 2013. The deployment of the 103 SDs was completed in March 2017, and to date about $70 \%$ of the array is in operation with a full triggering and DAQ system. Here we report an overview of the experiment, its capabilities and the technical details of the TALE SD trigger and DAQ system from 4 month's operation.

35th International Cosmic Ray Conference - ICRC2017

10-20 July, 2017

Bexco, Busan, Korea

${ }^{*}$ Speaker. 


\section{Introduction}

The Telescope Array (TA) experiment consists of the Surface Detector (SD) array of $700 \mathrm{~km}^{2}$ coverage and 38 fluorescence detectors (FDs), and it continues observations of ultra high energy cosmic rays with energies above $10^{18} \mathrm{eV}$ from 2008. In 2012 we started the Telescope Array Low Energy extension (TALE) experiment additionally installing 10 FDs pointing higher elevation angles, i.e., observing lower energy cosmic rays, than TA FDs. TALE FDs were located just beside the TA FD station at the north corner of the TA SD array, and the effective threshold energy of the experiment is successfully turned down to lower than $10^{16.5} \mathrm{eV}$. We report the cosmic ray energy spectrum in the wide energy range from $10^{15.9} \mathrm{eV}$ above $10^{20} \mathrm{eV}$ [四]. It has complicated structures showing several kinks and dips rather than a simple power law. We can see not only the steepening at $10^{19.6} \mathrm{eV}$ and the ankle at $10^{18.7} \mathrm{eV}$, but also a flattening at around $10^{16} \mathrm{eV}$ and a steepening at around $10^{17} \mathrm{eV}$.

In contrast with the ultra high energy region above $10^{18} \mathrm{eV}$ where the extragalactic cosmic rays are dominated, the galactic and the extragalactic components coexist in the lower energy range than $10^{18} \mathrm{eV}$. And the spectrum of extragalactic component has convolved information of source spectra, the spacial distribution of extragalactic sources, integration of energy losses during propagations and the shielding by the galactic magnetic field. Moreover, the galactic spectrum has a convolution of the physics process limiting the accelerated energy at galactic sources and the confinement of cosmic rays in the Galaxy.
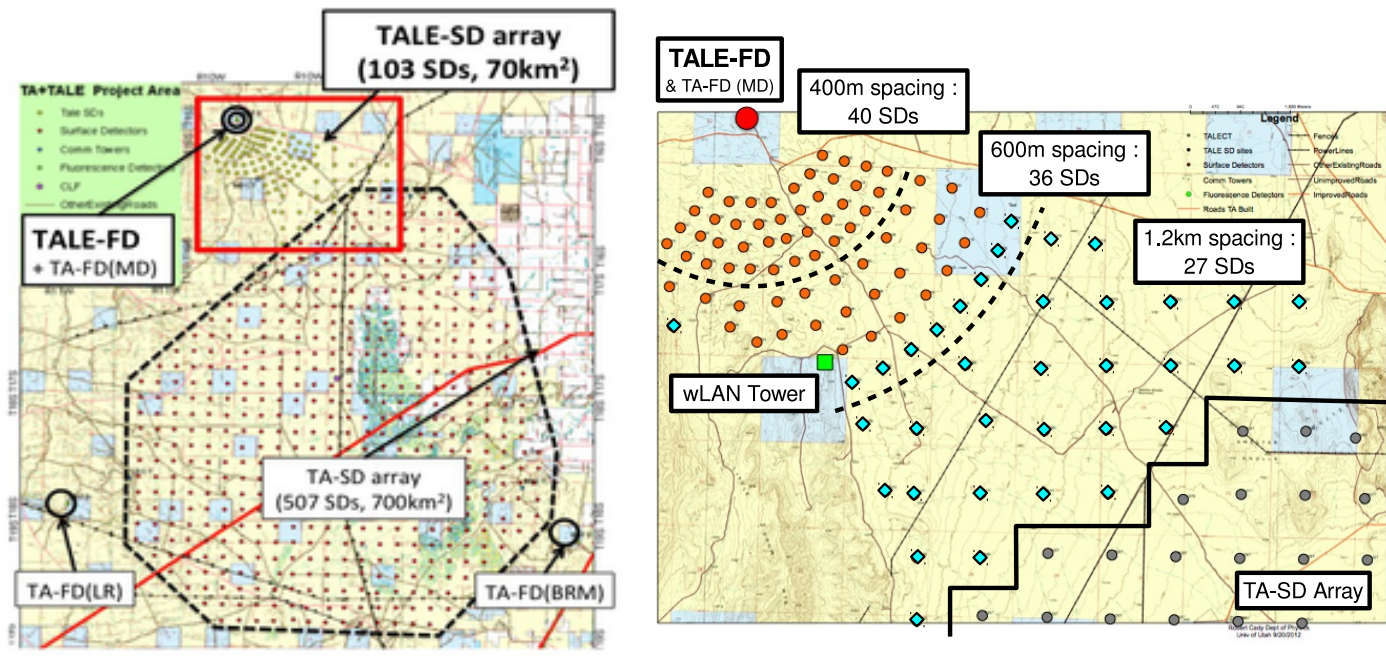

Figure 1: Left panel shows a map of the TA experiment site including TALE site. The small dots and the open circles indicate the TA SD counters and the TA FD stations, respectively. The close up of TALE site is shown in Right panel. All 103 counters were deployed, the circles show the SD counters including DAQ electronics. Other counters (rhombus) were deployed without electronics.

In order to resolve the convolved information the pressing issues are an energy spectrum measured with a high statistics and an energy-independent detection efficiency, arrival direction information measured with a constant exposure and a precisely measured chemical composition. For 
these precise measurements, we will construct the TALE hybrid detector with installing the air shower array of $70 \mathrm{~km}^{2}$ with 103 SDs in the FOV area of the TALE FDs as shown in Fig. $\mathrm{m}$.

The TALE FD station is located next to TA Middle Drum (MD) station, and these stations are watching almost same field of view in azimuthal angle. However, the TALE FD telescopes are watching higher in elevation than TA FDs, so that it can observe lower energy showers. The number of telescopes for TALE is 10, and they are looking 31 degree to 59 degree in elevation.

Fig. W shows a arrangement of the TALE SD array which has three different detector spacing. We deployed 40 SDs with $400 \mathrm{~m}$ spacing within the distance range of $3 \mathrm{~km}$ from the TALE FD station, and 36 SDs with $600 \mathrm{~m}$ spacing in the range from $3 \mathrm{~km}$ to $5 \mathrm{~km}$ from the TALE-FD station. In the connecting area between the high density area of TALE SD and the TA SD array, 27 detectors were deployed with $1.2 \mathrm{~km}$ spacing.

Based on simulation studies, the expected number of events by the SD array is 50,000 per year with the mode energy of $10^{16.5} \mathrm{eV}$, and the expected number of hybrid events is 5,000 per year with the mode energy of $10^{17.3} \mathrm{eV}$. This air shower array has $100 \%$ detection efficiency for energies above $1.4 \times 10^{17} \mathrm{eV}$, and hybrid observations with FDs provides a remarkable improvement in the Xmax determination achieving the error of $20 \mathrm{~g} / \mathrm{cm}^{2}$ comparing with the monocular FD resolution of $40 \mathrm{~g} / \mathrm{cm}^{2}$.

\section{The current status of the operation and the data analysis}

TALE FD telescopes had been installed in November 2012, and we continue operating since September 2013. We already reported the energy spectrum from $10^{15.9} \mathrm{eV}$ to $10^{20} \mathrm{eV} \mathrm{[?].}$

On the other hand, for the TALE SD array, the communication tower for the DAQ host was installed in Oct. 2012, and 35 SDs had been deployed at in the 400m spacing area. Among these, 16 detectors with the DAQ electronics which were originally manufactured as spares for TA SDs. The set of 16 SDs is making up an air shower array and the test DAQ system is continued with $100 \%$ efficiency from May 2013.

Fig. $\square$ is an example of a hybrid event taken by the TA-Middle Drum and TALE FDs together with the TALE SD array. On the left panel of Fig. B we show an energy distribution of the TALE FD events detected in 1080 hour observation time from June 2014 to March 2016. The right panel is a Monte Carlo calculated aperture. We also currently proceed with evaluations of our monocular event reconstruction accuracy comparing monocular analysis results with FD-SD hybrid analysis results for time matched events.

Mar. 2017, remaining SD counters were deployed and all of 103 counters had installed. 67 counters among them have new electronics and 16 old electronics were replaced with new one at the same time. Except some SDs because of initial failure (for example network trouble), renewaled DAQ system with new electronics has stably running with 55 SD counters.

\section{Development of New electronics for TALE/TAx4 SD}

We adopted a common design of new TALE SD and the TALE SD DAQ system with TAx4 SD in order to reduce development and production costs. We obtained funds (Grant-in-Aid for Scientific Research(S) from Japan Society for the Promotion of Science) to construct other 70 


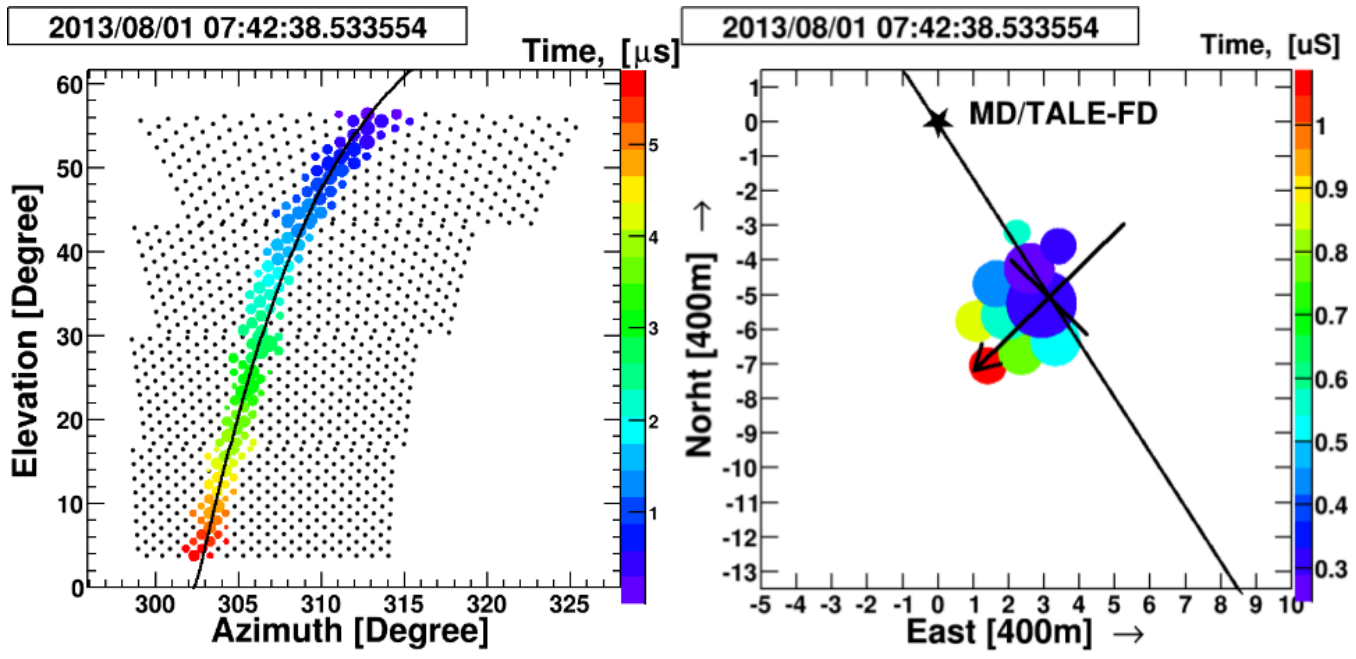

Figure 2: A hybrid event taken by the TA-Middle Drum and TALE FDs (Left) together with the TALE SD array (Right).
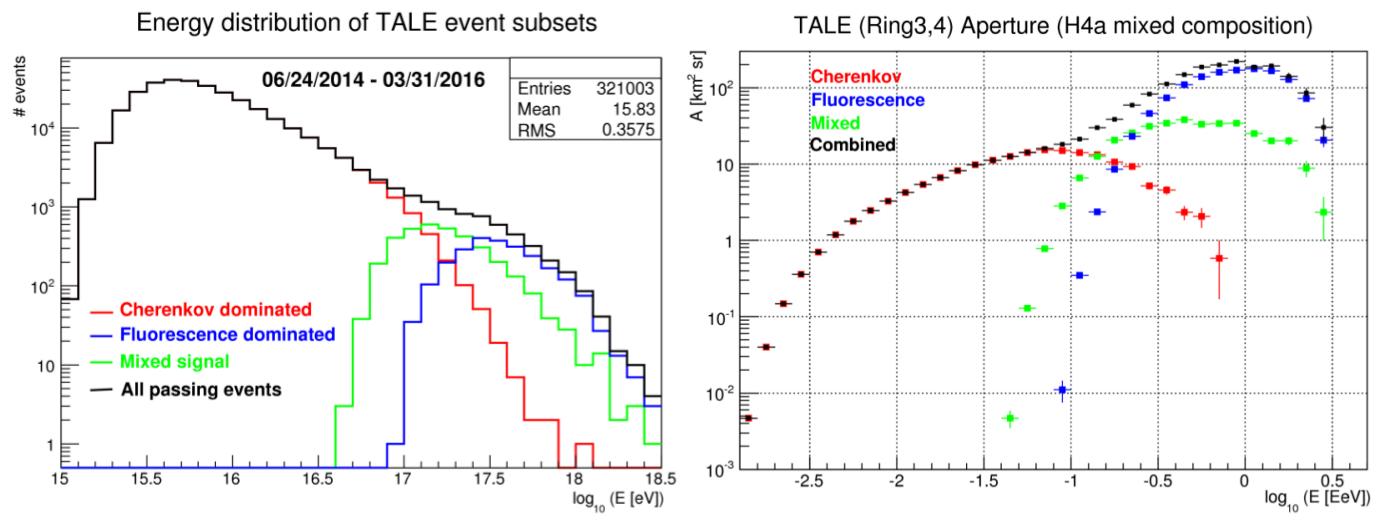

Figure 3: (Left) An energy distribution of the TALE FD events detected in 1080 hour observation time from June 2014 to March 2016. (Right)A Monte Carlo calculated aperture.

SDs and to continue the TALE experiment. The constructions and installations of the TALE SD array are scheduled to be finished in 2017 Japanese Fiscal Year and to start its full operation. The expected specifications of the TALE hybrid detector are as follows: the threshold energy is about $10^{16.5} \mathrm{eV}$; the expected event rate is about 5,000 hybrid events per year; the angular resolution reaches 1.0 degree; the Xmax resolution is $20 \mathrm{~g} / \mathrm{cm}^{2}$.

Today the wireless LAN modem used in the TA SD electronics is unavailable, so then the further production of the precise copy of the original is not possible. Then, we have designed a new electronics for TALE SD consisting of a new, available WLAN module (WiVicom, WVCWB$\mathrm{R}-022 \mathrm{~F}$ ), and as a result the protocol for the wireless LAN communications between the DAQ host and SDs has been changed from a specially designed one for the TA SD array to TCP/IP. With the introduction of the new protocol we can use a general-purpose personal computer as the DAQ host and an access point for broadcasting. In contrast to the new system, the existing system for TA SD 
array uses an SD electronics as the DAQ host consisting of a specially designed firmware.

The new electronics and its block diagram are shown in Fig. 田and Fig. [, respectively. With the introduction of the new WLAN module, the updates of the firmware for the FPGA and the software for the CPU were needed. Almost all of the development items for the new electronics and the DAQ system had been finished. Several field tests of the new electronics and the DAQ system had carried out in the TALE SD area. We have already confirmed the stability of the continuous run of the DAQ cycle for existing TALE SDs and the stability of long distance (max. $18 \mathrm{~km}$ ) communications.

One of the remaining major development items is the speeding up of the data transfer in SD electronics. The maximum number of SDs which a DAQ host can process is now 70. For example, adopting SPI instead of UART for the protocol between the wireless LAN module and the FPGA in the electronics, we expect $35 \%$ increase of the data transfer speed.

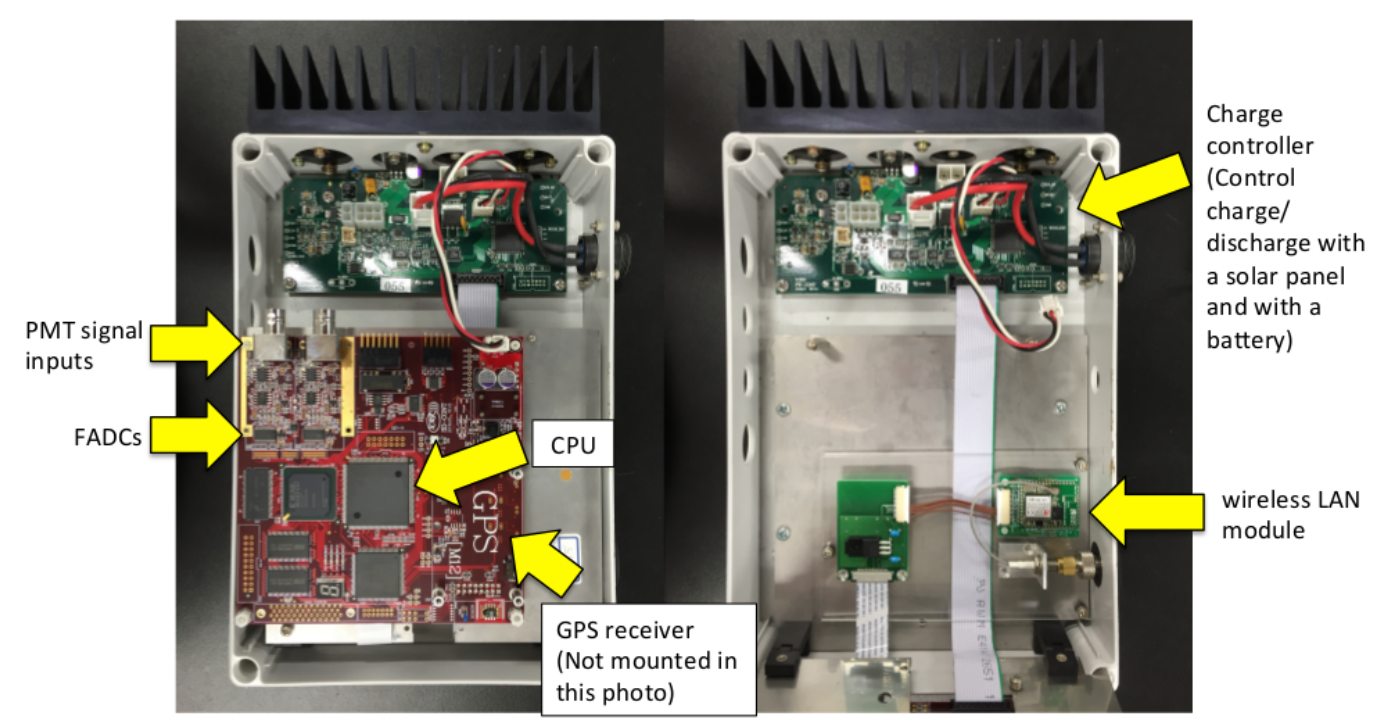

Figure 4: A picture of the new SD electronics for the TALE and TAx4 experiments.

\section{Summary}

TALE is an extension project of the TA experiment to lower the energy threshold down to $10^{16.5} \mathrm{eV}$. Construction of the hybrid detector with $10 \mathrm{FD}$ telescopes and an air shower array of $67 \mathrm{~km}^{2}$ with 103 SDs has mostly finished. The FDs and 16 SDs already installed were operated from 2013, a preliminary results of FD mono energy spectrum was reported in this conference. SD array data analysis has been also developing, therefore hybrid analysis will be started soon.

The new SD electronics and new DAQ system for TALE and TAx4 have been developed. The production of 100 sets and installation of 70 sets of new electronics have already finished. Although some more improvements of DAQ system are required for the TALE SD array of 103 SDs, also TAx4 SD array, new DAQ system is stably running with 55 SDs. 


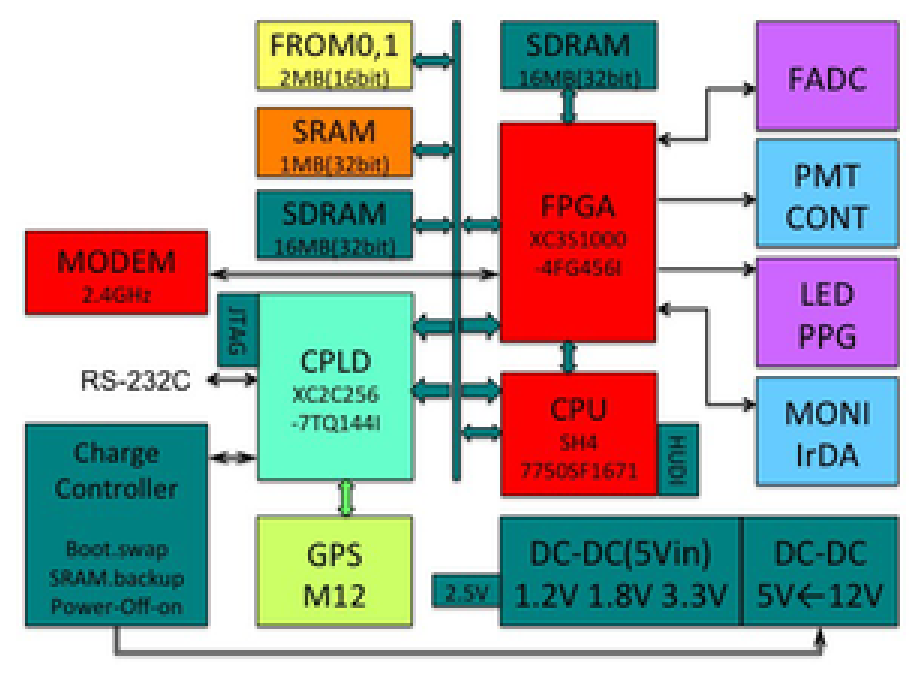

Figure 5: A block diagram of the new SD electronics.

\section{Acknowledgements}

The Telescope Array experiment is supported by the Japan Society for the Promotion of Science through Grants-in-Aids for Scientific Research on Specially Promoted Research (15H05693) and for Scientific Research (S) (15H05741), and the Inter-University Research Program of the Institute for Cosmic Ray Research; by the U.S. National Science Foundation awards PHY-0307098, PHY-0601915, PHY-0649681, PHY-0703893, PHY-0758342, PHY-0848320, PHY-1069280, PHY1069286, PHY-1404495 and PHY-1404502; by the National Research Foundation of Korea (2015R1A2A1A01006870，2015R1A2A1A15055344， 2016R1A5A1013277，2007-0093860, 2016R1A2B4014967); by the Russian Academy of Sciences, RFBR grant 16-02-00962a (INR), IISN project No. 4.4502.13, and Belgian Science Policy under IUAP VII/37 (ULB). The foundations of Dr. Ezekiel R. and Edna Wattis Dumke, Willard L. Eccles, and George S. and Dolores Doré Eccles all helped with generous donations. The State of Utah supported the project through its Economic Development Board, and the University of Utah through the Office of the Vice President for Research. The experimental site became available through the cooperation of the Utah School and Institutional Trust Lands Administration (SITLA), U.S. Bureau of Land Management (BLM), and the U.S. Air Force. We appreciate the assistance of the State of Utah and Fillmore offices of the BLM in crafting the Plan of Development for the site. We also wish to thank the people and the officials of Millard County, Utah for their steadfast and warm support. We gratefully acknowledge the contributions from the technical staffs of our home institutions. An allocation of computer time from the Center for High Performance Computing at the University of Utah is gratefully acknowledged.

\section{References}

[1] T. AbuZayyad et. al, in this conference 